\title{
A Web Based Automated Tool for Course Teacher Evaluation System (TTE)
}

\author{
Mahfida Amjad ${ }^{\mathrm{a}}$, Nusrat Jahan Linda ${ }^{\mathrm{a}}$ \\ ${ }^{a}$ Stamford University Bangladesh, Dhaka, Bangladesh
}

Received: 02 November 2019; Accepted: 14 February 2020; Published: 08 April 2020

\begin{abstract}
For any educational institution course or teacher evaluation is an integral part for the betterment and effective education system. Student's feedback could be taken as one of parts of teacher evaluation. This paper has tried to evaluate the effectiveness of the course teacher evaluation system from the students' feedback of their corresponding courses. At Stamford University Bangladesh currently the teacher's evaluation task is running by manually which is very time consuming, slow and a lengthy process. It also needs number of human resources for completing this task. This paper has presented a web based automated tool for Course Teacher Evaluation System (TTE). In this technique student's opinions are taken from some predefined questions in a web based platform for evaluating a teacher of any particular course. And the result from the data analysis is automatically generated along with a graphical representation. From the generated report it becomes very easy for the teacher to understand and focus on the area where they need to emphasize for their personal and professional growth. As the results are generated automatically from the survey it saves time as well as man power.
\end{abstract}

Index Terms: Web based automated tool, course teacher evaluation system, student's feedback, survey, automated graph.

(C) 2020 Published by MECS Publisher. Selection and/or peer review under responsibility of the Research Association of Mode rn Education and Computer Science

\footnotetext{
* Corresponding author. Mahfida Amjad

E-mail address: mahfidaamjad@stamforduniversity.edu.bd.
} 


\section{Introduction and Literature Review}

Evaluation is a process of making judgments over a level of performance or achievement by assessing, testing and measuring. The main purpose of course teacher evaluation is personal and professional growth that leads to improved performance in any educational system. The newly appointed teachers should be able to accelerate the learning curve that comes with their new job. Proper assistance and training can be provided to contending teachers for guiding them to improve their mastery of content delivery, manage classrooms; enhance student engagement and participation, motivation, and learning. Teacher observations and post-observation conferences should occur at regular intervals throughout the year and result in more consistent and timely feedback about their performance [2].

Student's feedback is considered as the most effective and reliable method for teacher's evaluation [1]. Evaluating teacher's performance is essential to improve learning system as it is related to student's learning. As a result, teacher's performance should be assured through the proper use of teacher evaluation technique. This technique refers to the formal process for any educational institution to review and rate teachers' performance and effectiveness in the classroom. The responses from these evaluations are used to provide feedback to teachers and guide their professional development [3].

In Stamford University Bangladesh it uses an evaluation system which is manual or hardcopy based. For doing the evaluation the students are asked to fill up a form of 10 questions. After the survey all the forms are collected and the data analysis is done by manually using Microsoft Excel which is very lengthy and time consuming matter. And from this concern the idea of a web based automated system is generated to make this evaluation system easier and faster compared to the manual system web based automated tool for Course Teacher Evaluation System (TTE) it is possible to analysis the data00automatically after taking the survey along with an auto generated graph showing the minimum and maximum values for corresponding questions. From this graph it is easily understandable to a particular teacher where he or she should focus or give more emphasize to improve him/her.

The rest of this paper is organized as follows; Section 2 discusses the literature review. Section 3 and 4 illustrates the methodology, data analysis and result respectively. Finally Section 5 concludes this paper with discussion and conclusion.

\section{Literature Review}

Basic concept and feature of TTE along with its advantages has been discussed in this section.

\subsection{Background Study}

The main objective of evaluation of any system is assessing, testing and measuring which is done for qualitative improvement. It is a process of judgments over a level of performance. The aim of this study is to improve the quality of teaching by introducing student's feedback in an educational institution.

Starting during the 1990s and through the 2000s, examinations of year-to-year understudy test information reliably indicated that a few educators helped their understudies adapt altogether more than did different instructors. Backing bunches contended that present quality-control frameworks for instructors were inadequate. In a persuasive 2009 report, TNTP (some time ago the New Teacher Project), found that in excess of 99 percent of educators in the 12 regions it considered were positioned good on assessments. Generally, instructor assessment frameworks depended vigorously on homeroom perceptions directed by principals or other school directors. But many systems have undergone significant changes in recent years [4]. 
With the most recent advances in substance and guidance recognize and assets promptly accessible, experienced instructors will be better prepared to overcome the impacts of burnout. They will be bound to sink into an effective and fulfilling profession with the help of an evaluation system [5].

\subsection{TTE System}

The TTE will be tends to be utilized for an educator advancement. Assessment results can be utilized as proof for expelling a tenured educator for poor performance.

\subsubsection{Advantages of TTE}

- The primary purpose of teacher evaluation is personal and professional growth that leads to improved performance.

- Administrators will also be benefited by teacher evaluation system.

- New educators ought to have the option to quicken the expectation to learn and adapt that accompanies their new position. Directed help and preparing ought to be given to battling educators, helping them improve their authority of substance and instructional method; upgrade understudy commitment, inspiration, and learning.

- Teacher observations and post-observation conferences should occur at regular intervals throughout the school year and result in more consistent and timely feedback about their performance.

- The improvement of individual educators after some time ought to be guided by individualized proficient advancement and development plans.

\section{Methodology}

In this TTE system for evaluating teacher students have to complete their registration into this system. This registration form consists of student email id, student ID, batch, section, course title, course code and teacher name. Table 1 shows the attributes of the student registration process. Fig. 1 shows the use case diagram of student and Fig. 2 Student registration form of TTE.

TABLE 1. ATTRIBUTES OF THE STUDENT REGISTRATION PROCESS

\begin{tabular}{|c|}
\hline Email address \\
\hline Student ID \\
\hline Batch \\
\hline Course Section \\
\hline Course Code \\
\hline Course Title \\
\hline Course Teacher \\
\hline
\end{tabular}

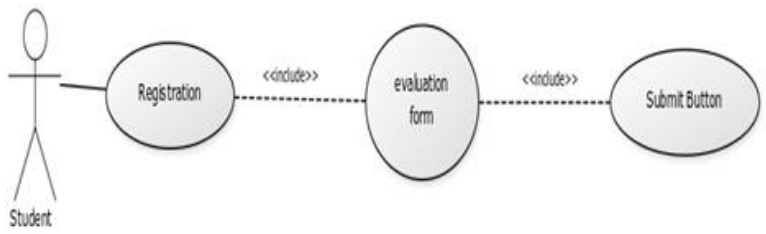

Fig. 1. Use case diagram of student 


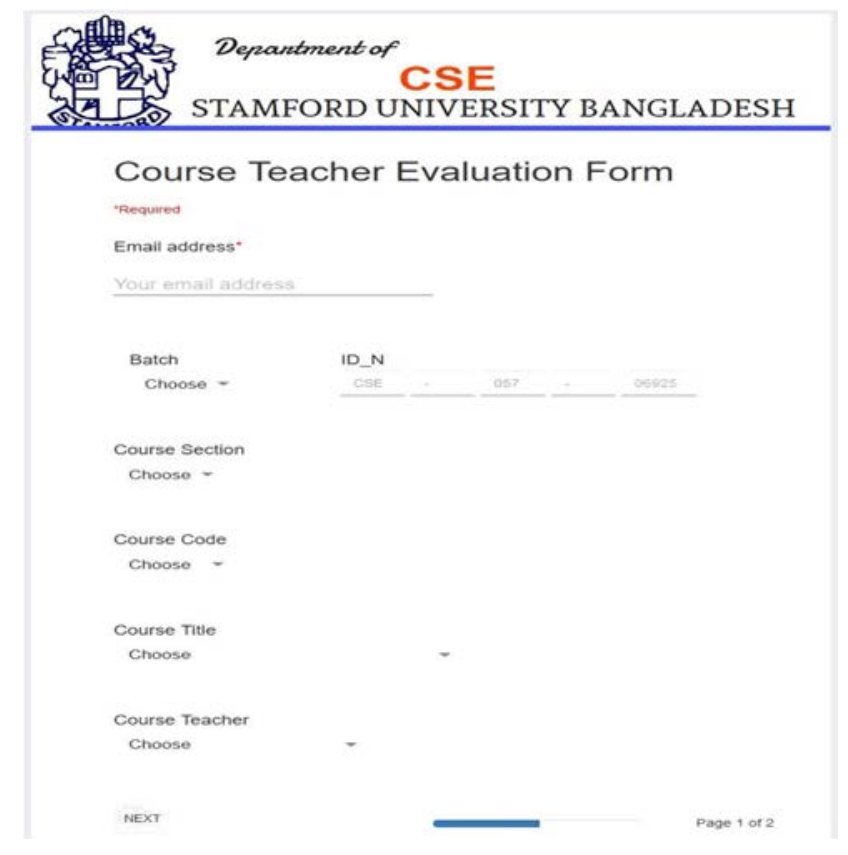

Fig. 2. Student Registration Form

Table 2 shows the 10 questions about the rules and regulation of the university along with the teacher performance in the class room. A short discussion about the above questions is stated as follows:

Q. 1: Course teacher provided course outline at the start of the trimester:

A teacher should give a course outline of his/ her subject in his/her first class. It helps the students to understand how they should read and what the topics of that course are.

Q. 2: Were exams, quizzes and assignments relevant to the course?

It is another important fact that exams, quizzes and assignments are relevant to the course or not. If this criterion is maintained then students will be interested in class lecture. They will focus what their teacher asks and will try their best to understand the topics for doing best in the exam [14].

Q. 3: How well the teacher was innovative and interesting to make the lessons interactive?

Innovation in education encourages the students to explore, research and use all the modern tools to discover something new. It involves a different way of looking and thinking at complex problems and solving them. The thinking process that goes into it will help students develop their creativity and their problem solving and analytical skills. So a teacher must be innovative and interesting to make the lessons interactive [15].

Q. 4: Regularity in starting and ending class on time:

If a teacher arrives in class on time he/she can complete the lecture properly. As a result before exam the syllabus will be complete and it will also help to complete the quizzes on time. So being present in class on time is very crucial and also a good practice for a teacher [6].

Q. 5: The teacher was cooperative and willing to give you time for counselling:

In a particular class all the students may not be able to grasp the topic equally. Some students may be weak to understand the lecture in class time. So they need more attention. For their improvement teacher should give them a counselling hour so that all students can learn equally [7]. 
TABle 2. Survey Questions Of Scores From 1-5 Which Means As 5: Excellent; 4: Very Good; 3: GoOd; 2: Average; 1: Below AVERAGe.

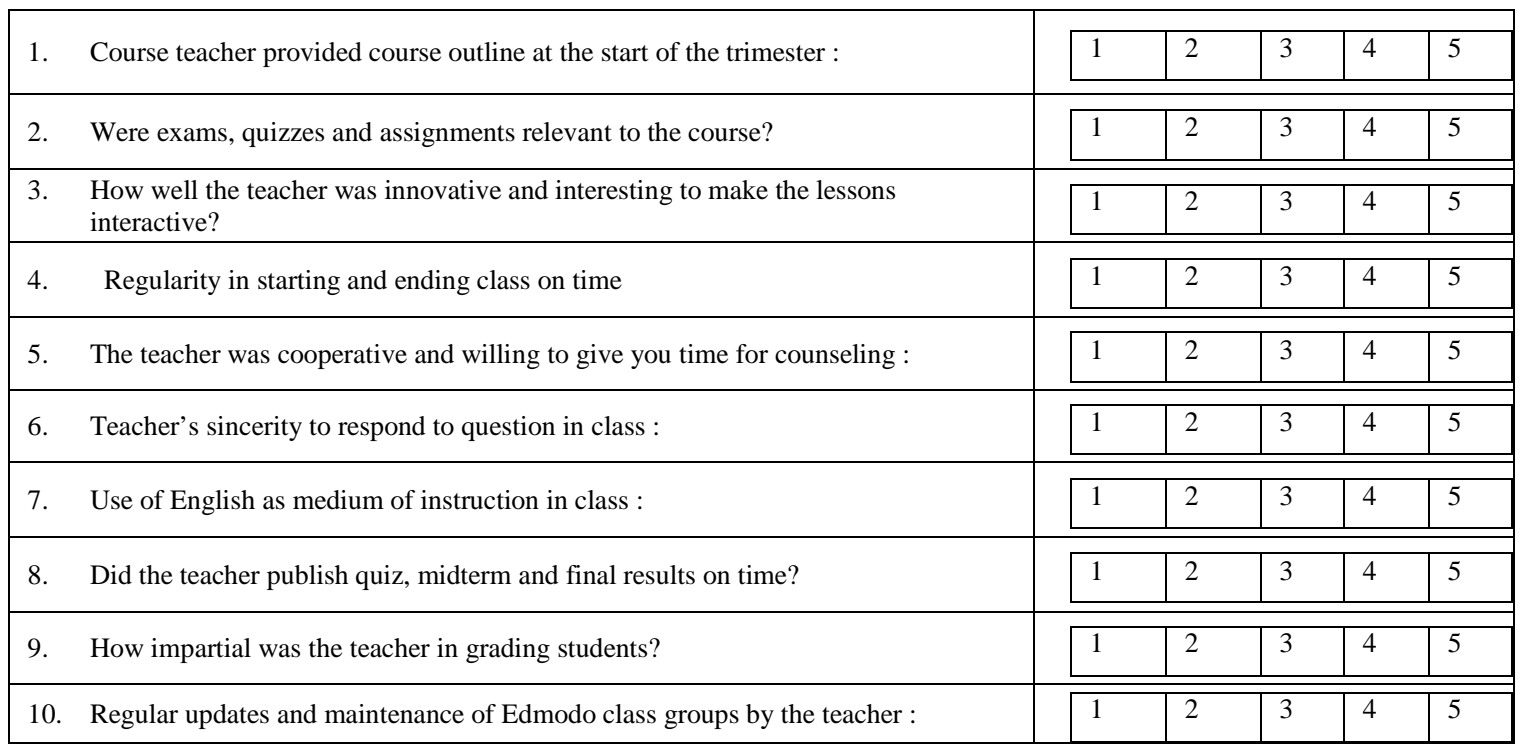

Q. 6: Teacher's sincerity to respond to question in class:

The teacher should respond to student's questions accordingly. He/she should try to give the correct answer and explain it. By doing this the students can get the clear idea about the topic [8].

Q. 7: Use of English as medium of instruction in class:

The medium of instruction is the language used by the teacher to teach. It will be easy for the students if the teacher teach in English in the class as their books are written in English. It will increase the amount of exposure of the learners and the opportunities to communicate and also to develop their control of it [9].

Q. 8: Did the teacher publish quiz, midterm and final results on time?

Teacher should publish quiz, midterm and final results on time which will make them more sincere, active and also be interested in their lesson [10].

Q. 9: How impartial was the teacher in grading students?

The teacher should be impartial in their grading system. He/she does not play favorites among the students and cannot allow their emotion into their discipline [11].

Q. 10: Regular updates and maintenance of Edmodo class groups by the teacher:

Stamford University Bangladesh uses Edmodo which is a global education network where teachers can manage their classroom, engage their students, send messages, share class materials, and make learning accessible anywhere. So it is necessary for the teacher to be updated through the edmodo class group [12].

The course teacher evaluation form of TTE has been shown in Fig. 3. 


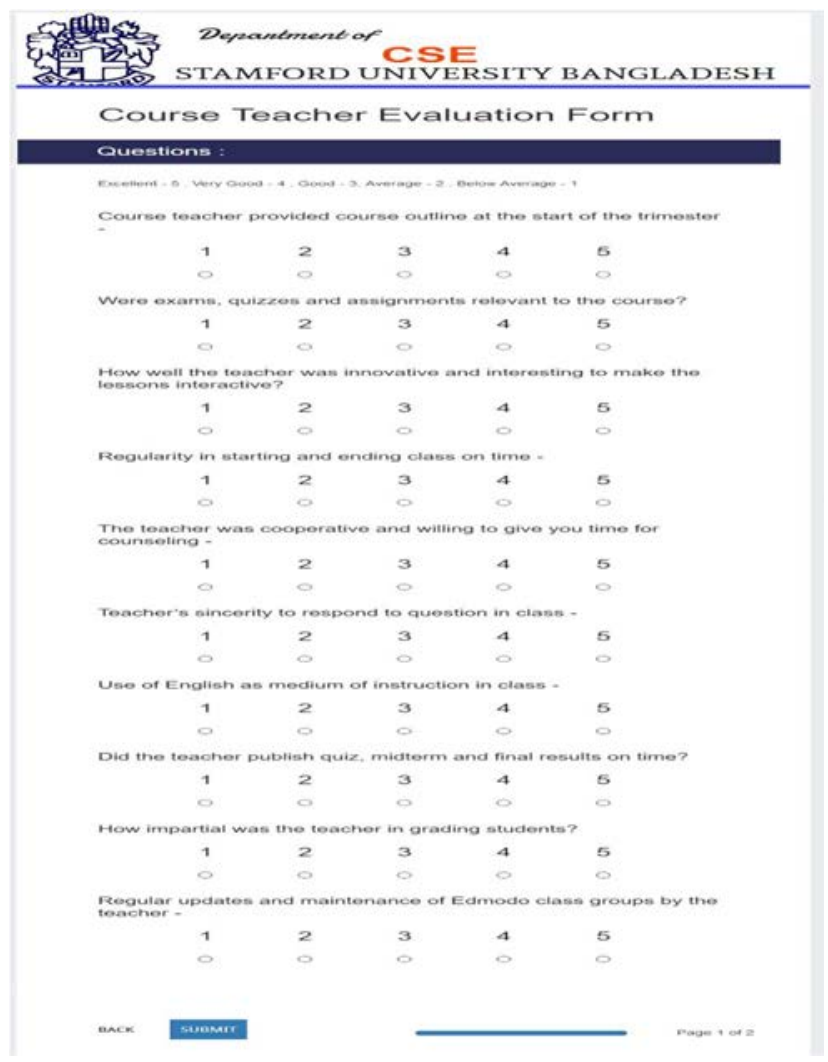

Fig. 3. Course Teacher Evaluation Form of TTE

\section{Data Analysis and Result}

In TTE system the main control is done by the admin. Initially the admin has to sign up for creating an account. This account is created by an email and password. This password should have at least 8 characters, one character must be capital letter, one must be small letter and one must be digit. This process helps to create strong password.

Fig. 4 shows the interface of admin signup and login of TTE. If the admin already have an account, they do not need to sign up again. They can just use login button and use their registered username, password. To view the result or evaluation of the completed survey the admin needs to select the teacher name, batch no, course, section from the list. Table 3 shows the attributes of the admin whereas Fig. 5 shows the use case diagram of the admin.

TABLE 3. ATTRIBUTES OF THE ADMIN

\begin{tabular}{|c|}
\hline User Name \\
\hline Email Address \\
\hline Password \\
\hline
\end{tabular}




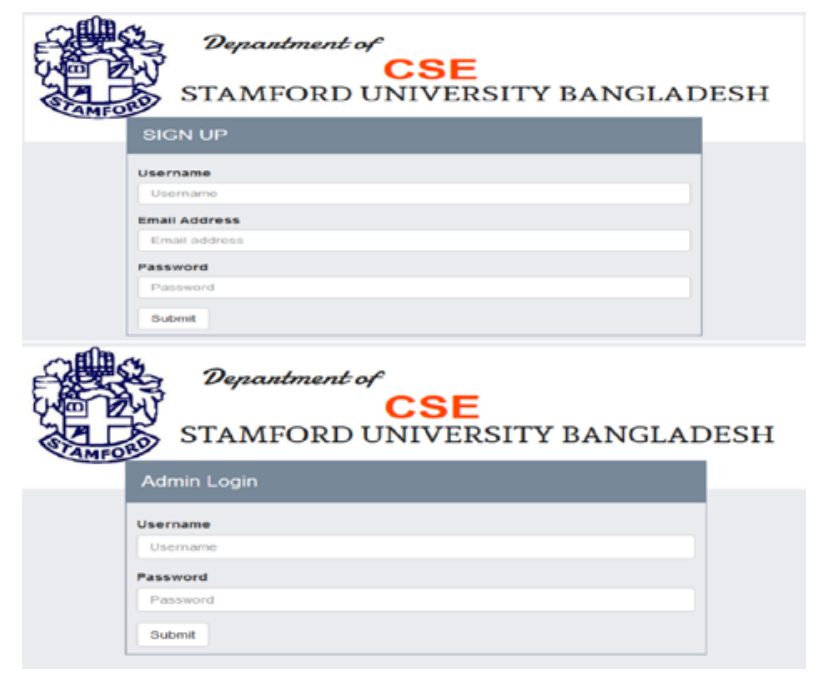

Fig. 4. Admin Sign up and Login Interface of TTE

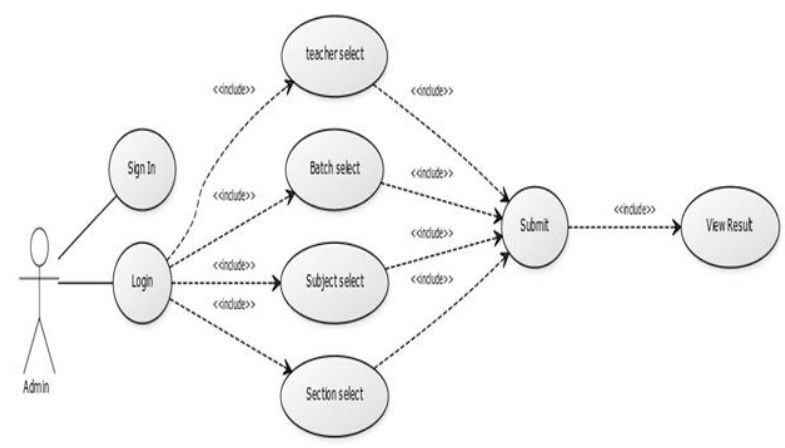

Fig. 5. Use case diagram of admin

After selecting all the necessary fields the admin will be able to view the result like the Fig. 6 . It shows the average value of the corresponding 10 questions and based on the value the automated graph will be generated like in Fig. 6.

\section{Discussion and Conclusion}

The main feature of the TTE is to create the automated graph. Teacher can see their evaluation of their course for any particular batch and section. This system gives a full report to the teacher about their course. In this report teacher can see their average marks of the 10 questions with graph which helps them to identify the area where they need to more focus. The TTE is a fully automated web based system which saves time to evaluate the courses very easily. It is also possible to save the result as pdf file format and print as a report. 
The web based TTE system will be very effective for any educational institution. From this system a teacher can easily understand in which area they needs to improve. It helps the teacher's personal and professional growth that leads to better performance.

This web based tool works only in computer system. In near future this system will be moved to an advanced level by making a mobile application which will be implemented in android OS initially.

\section{Acknowledgements}

The authors wish to thank Dr. Kamruddin Nur for his support and guidance. This work was supported in part by Stamford University Bangladesh.

\section{References}

[1] Musharraf Husain, Sabina Khan, Students’ feedback: An effective tool in teachers’ evaluation system, Departments of Surgery, 1Pathology, Adjunct Faculty MEU, Hamdard Institute of Medical Sciences and Research, Jamia Hamdard, New Delhi, indi India, January 24, 2017.

[2] D Stufflebeam, New directions for evaluation, Wiley Online Library, 2001.

[3] Talal Almutairi, Durham University, UK Peter Tymms, The Tools of Teacher Evaluation: What Should Be Used in Teacher Evaluation from the Teachers' Perspective, International Business \& Education Conferences, London, United Kingdom June 7-11, 2015.

[4] Margaret K Snooks, Using Practice Tests on a Regular Basis to Improve Student Learning, New Directions for Teaching and Learning, 2004(100):109 - 113.

[5] Janelle Cox, 10 ways to keep your class Interesting, ThoughtCo, August 16, 2019.

[6] Teny Shapiro, The Educational Effects of School Start Times, DOI: 10.15185/izawol.181, September, 2015.

[7] Dana Edwards, The school counselor's role in helping teachers and students belong, Elementary School Guidance \& Counseling, Vol. 29, No. 3 February 1995, pp. 191-197.

[8] Kathleen Listman, How do you deal with a sincere question in the classroom that avoids embarrassment, M.Ed University of Texas, Arlington, Jul 10, 2018.

[9] Ameesha Wijayantunga (September, 2019), English as a Medium of Instruction in Secondery Schools In Srilanka: Challenge, International Conference on Education, September 2018, DOI: 10.17501/icedu.2018.4117.

[10] Melissa Kelly, Pretests Effective Tool to Target Instruction, ThoughtCo, January 16, 2019.

[11] Rema N.Hanna and Leigh L.Linden, Discrimination in Grading, American Economic Journal: Economic Policy, Vol. 4, No. 4 (November 2012), pp. 146-168.

[12] Salubsri Charoenwet and Ami Christensen, The Effects of Edmodo Learning Network on Students Perception's Self Regulated Learning Behaviors and Learning Performance, Proceedings of The 10th International Multi-Conference on Society, Cybernetics and Informatics, IMSCI 2016.

[13] Guri Skedsmo, Stephan Gerhard Huber Teacher evaluation the need for valid measures and increased teacher involvement, Educational Assessment Evaluation and Accountability 30(2), DOI: 10.1007/s11092-018-9273-9, February, 2018.

[14] Santiago, P. \& Benavides, F., Teacher Evaluation: A Conceptual Framework and Examples of Country, International Journal of Evaluation and Research in Education (IJERE), 2019.

[15] Little, O, Goe, L. \& Bell, C., A Practical Guide to Evaluating Teacher Effectiveness, The Journal of Effective Teaching, 2009. 


\section{Author's Profile}

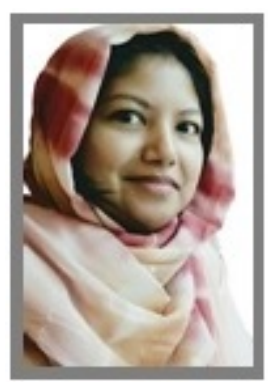

Mahfida Amjad was born in 1985 in Dhaka, Bangladesh. She has completed her Master degree in Information Technology from Institute of Information Technology from University of Dhaka in 2009. And she completed B.Sc. in Computer Science \& Engineering from Manarat International University in 2007. She is a faculty member of Computer Science and Engineering (CSE) Department of Stamford University Bangladesh. She has devoted herself in teaching profession since 2012. Her research area is wireless ad hoc network, deep learning, IoT based automation.

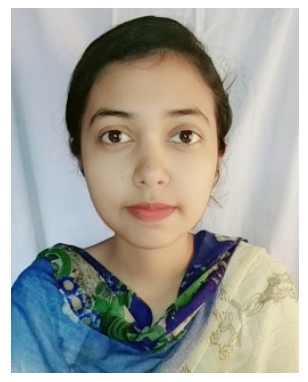

Nusrat Jahan Linda was born in 1997 in Bangladesh. She has completed B.Sc in Computer Science and Engineering from Stamford University Bangladesh in 2019. Currently she is working on web based automated system.

How to cite this paper: Mahfida Amjad, Nusrat Jahan Linda. "A Web Based Automated Tool for Course Teacher Evaluation System (TTE)", International Journal of Education and Management Engineering(IJEME), Vol.10, No.2, pp.11-19, 2020.DOI: 10.5815/ijeme.2020.02.02 